Please do not remove this page

RMIT

UNIVERSITY

\title{
RF photonic instantaneous frequency measurement using DC photo-detection
}

Emami, Hossein; Sarkhosh, Niusha; Bui, Lam; Mitchell, Arnan

https://researchrepository.rmit.edu.au/esploro/outputs/9921864069901341/filesAndLinks?institution=61RMIT_INST\&index=null

Emami, H., Sarkhosh, N., Bui, L., \& Mitchell, A. (2008). RF photonic instantaneous frequency measurement using DC photo-detection. Proceedings from the Joint Conference of the Opto-Electronics and Communications Conference 2008 and the 2008 Australian Conference on Optical Fibre Technology OECC/ACOFT 2008, 1-2. https://doi.org/10.1109/OECCACOFT.2008.4610443

Published Version: https://doi.org/10.1109/OECCACOFT.2008.4610443

Repository homepage: https://researchrepository.rmit.edu.au (C) 2008 Engineers Australia and the Australian Optical Society

Downloaded On 2023/04/26 21:22:36 +1000 


\title{
RF Photonic Instantaneous Frequency Measurement Using DC Photo-Detection
}

\author{
H. Emami, N. Sarkhosh, L. A. Bui, A. Mitchell \\ School of Electrical and Computer Engineering, RMIT University \\ GPO Box 2476V, Melbourne, Victoria, 3001, Australia \\ Phone: +61 3 99253250, Fax: +61 399252007 \\ Email: hossein.emami@rmit.edu.au
}

\begin{abstract}
A microwave photonic Instantaneous Frequency Measurement (IFM) system based on a photonic transversal approach and DC-detection is proposed and practically demonstrated. This system is able to measure the RF frequency and power level independently.

\section{Introduction}

Instantaneous Frequency Measurement (IFM) receivers are important parts of Electronic Warfare systems. These receivers identify the major frequency of the potential threat and therefore must act instantaneously.

Electrical IFM receivers have been in use for many years [1]. They are essentially an interferometer formed by delay lines and RF couplers. These devices can be bulky and band-width limited due to unwanted radiation, $\mathrm{RF}$ attenuation and high frequency dispersion [2].

In recent years, microwave photonics has been explored as a means to overcome band-width limitations [3-6]. Microwave photonics has been used for frequency measurement. One solution uses dispersion to achieve wavelength dependent delays; however broad-band photo-detectors are required which can be expensive [4].

In this paper, we demonstrate a method with DC output which can decrease the total cost of the system.
\end{abstract}

\section{IFM Concept}

Fig.1 presents a diagram of an IFM system where two orthogonal DC signals are obtained from the input RF signal. The RF signal is divided into two equal portions. Each portion feeds an interferometer formed by a power divider, delay lines and a mixer. The lower interferometer includes a $90^{\circ}$ phase shifter to produce an orthogonal output component. The interferometer outputs have two orthogonal DC components $\left(V_{\text {out } 1}, V_{\text {out } 2}\right)$ which can be used to measure both frequency and RF power independantly. Having conceived an IFM system, we are now able to implement this concept in the optical domain.

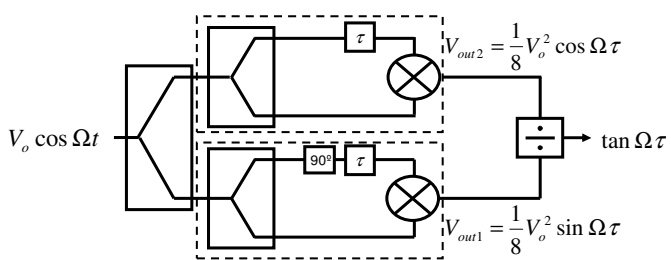

Fig. 1 The block diagram of an IFM system

\section{Photonic IFM}

In the RF domain, a $90^{\circ}$ phase shift can be achieved using hybrid couplers; however these devices have limited bandwidth. A broad-band $90^{\circ}$ phase shift, can be achieved using a photonic transversal approach[6]. The schematic diagram of such a system is shown in Fig. 2.

An RF signal is divided by two equal portions using a Wilkinson power divider. The first portion feeds a $2 \times 1$ Mach-Zehnder Modulator (MZM1) biased at quadrature. Carriers $\lambda_{\mathrm{o}}$ and $\lambda_{2}$ are combined using a $3 \mathrm{~dB}$ coupler and input to the first port of MZM1. Carrier $\lambda_{1}$ is input into the second port (and thus modulated with an inverted signal). The effective modulations are shown in Fig. 2 inset (a). The modulated carriers are then input to a 3port optical circulator. Port2 of the circulator is connected to a cascaded grating which is used to achieve a wavelength dependant delay. The delayed carriers are output from Port3 of the circulator and input to MZM2.

The RF input to MZM2 is the $2^{\text {nd }}$ portion of the RF signal to be measured which has been delayed using a length of co-axial cable. The twice modulated carriers are then amplified by an Erbium Doped Fibre Amplifier (EDFA) and input to a Wavelength Division Multiplexer (WDM) separating all wavelengths. Carriers $\lambda_{1}$ and $\lambda_{2}$ are combined to produce the two-tap transversal filter (Fig. 2 inset (b)), while carrier $\lambda_{\mathrm{o}}$ remains separated and acts as a reference (Fig. 2 inset (c)). Both signals are then detected, DC-filtered and logged.

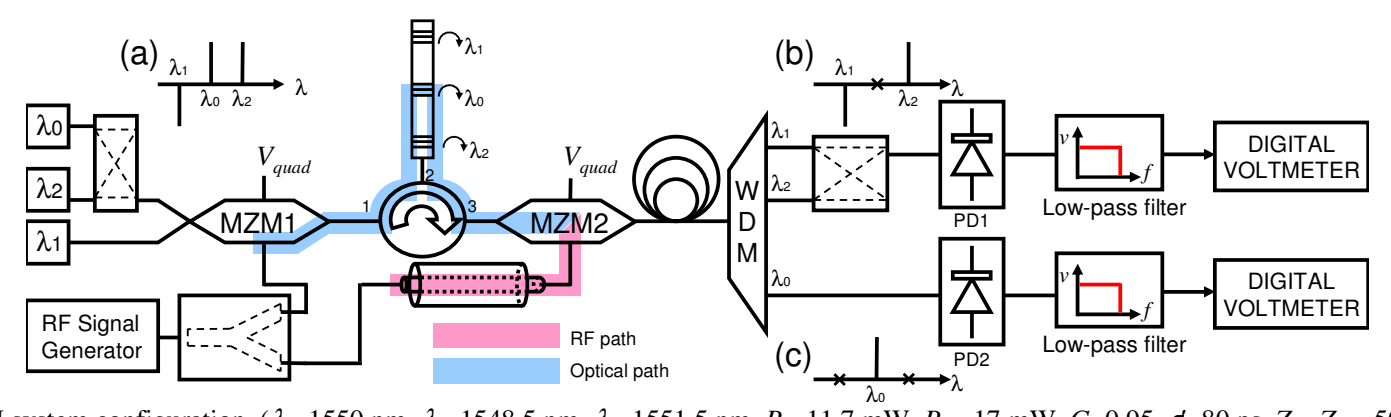

Fig. 2 IFM system configuration. ( $\lambda_{0}=1550 \mathrm{~nm}, \lambda_{1}=1548.5 \mathrm{~nm}, \lambda_{2}=1551.5 \mathrm{~nm}, P_{0}=11.7 \mathrm{~mW}, P_{90}=17 \mathrm{~mW}, G=0.95, \tau^{\prime}=80 \mathrm{ps}, Z_{i n}=Z_{P D}=50 \Omega, V_{\pi}=5 \mathrm{v}$.) 
Having a two-tap transversal filter with a reference in between, it should be possible to have two orthogonal measurements to be used for frequency measurement.

The DC signals present at the lower and upper arms can be described as:

$$
\begin{gathered}
V_{D C 0}=\alpha_{0}+\beta_{0} P_{R F}+\gamma_{0} P_{R F} \cos \varphi(\Omega) \\
V_{D C 90}=2 \alpha_{90}+2 \beta_{90} P_{R F}+2 \gamma_{90} P_{R F} \sin \Omega \frac{\tau^{\prime}}{2} \sin \varphi(\Omega)
\end{gathered}
$$

where $\Omega$ and $\mathrm{P}_{\mathrm{RF}}$ are the angular RF frequency and power, $\tau$ is the delay between $\lambda_{1}$ and $\lambda_{2}$, and $\varphi(\Omega)$ is the phase response of the RF path relative to the Optical path as shown in Fig. 2. The parameters $\alpha_{0}, \beta_{0}$, and $\delta_{0}$ are constants defined as below:

$$
\alpha_{0}=\frac{1}{2} G Z_{P D} P_{0}, \beta_{0}=\frac{1}{8} G Z_{P D} P_{0} \frac{\pi^{2}\left(1+M^{2}\right) Z_{\text {in }}}{2 V_{\pi}^{2}}, \delta_{0}=\frac{\pi^{2}}{2 V_{\pi}^{2}} G M Z_{P D} Z_{\text {in }} P_{0}
$$

where $G$ is the total link gain, $Z_{P D}$ and $Z_{i n}$ are the photodetector output impedance and MZMs input impedance respectively. $V_{\pi}$ is the MZMs quadrature voltage, and $M(\Omega)$ is the absolute magnitude response of the RF path. $P_{0}$ is the optical power of carrier $\lambda_{\mathrm{o}} . \alpha_{90}, \beta_{90}$, and $\delta_{90}$ can also be defined in the same way by assuming the same optical power for carriers $\lambda_{1}$ and $\lambda_{2}$.

From Eq. (1) and (2), it can be deduced that:

$$
\frac{V_{D C 0}-\alpha_{0}}{V_{D C 90}-2 \alpha_{90}}=\frac{\beta_{0}+\gamma_{0} \cos \varphi(\Omega)}{2 \beta_{90}+2 \gamma_{90} \sin \Omega \frac{\tau^{\prime}}{2} \sin \varphi(\Omega)}
$$

Eq. (3) establishes a unique relationship between RF frequency and system parameters and can thus predict the frequency of an RF signal independent of power.

To asses the IFM system of Fig. 2, the system parameters were set as defined in the caption of Fig. 2. Four different RF power levels $\left(P_{R F}=3,6,9,12 \mathrm{dBm}\right)$ were used to obtain four sets of measurements.

Fig. 3 shows the measurements obtained from the digital voltmeters along with the prediction results from Eq. (1) and (2). Good agreement is evident. Due to oscillatory nature of Eq. (1) and (2), there are nine distinct bands within which frequency measurement is unambiguous. These measurement sets were then used to predict RF frequency using Eq. (3) as shown in Fig. 4 a). The RF power level is also predicted and shown in Fig. 4 b). Good agreement between prediction and measurement is evident. Some deviations can be observed at the ends of each band due to zero gradients at peaks and nulls. Some inconsistencies occurred above $9 \mathrm{GHz}$ due to co-axial cable attenuation and multi-mode dispersion. The system band-width could be increased by integration using, for instance, broad-band travelling wave co-planar transmission lines in place of the coaxial cable.
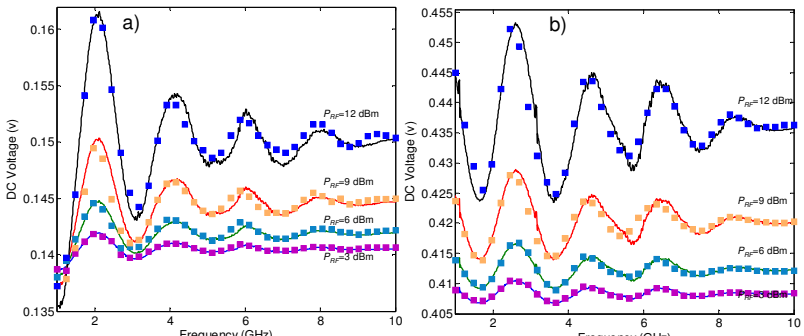

Fig. 3 Measured and predicted results for a) reference tap, b) two-tap transversal filter
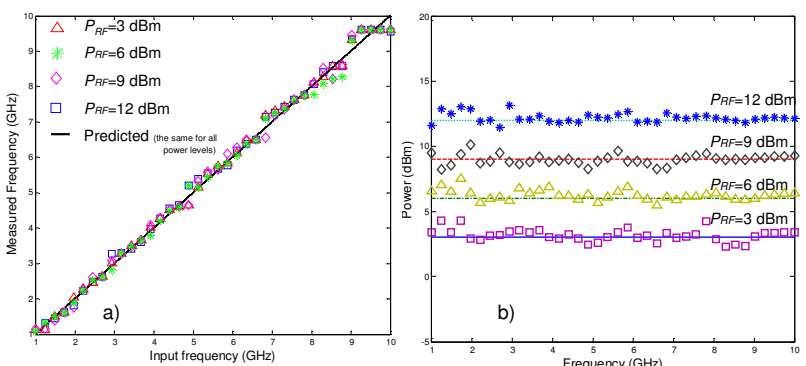

Fig. 4 Measured and predicted a) RF frequency b) RF power level

\section{Conclusion}

A broad-band IFM system based on a photonic approach is proposed and demonstrated. As the frequency measurement is based on DC signals, low-cost, lowfrequency photo-detectors can be employed to decrease the total cost of the system.

\section{References}

1. H. Gruchala, and M. Czyzewski, "The instantaneous frequency measurement receiver in the complex electromagnetic environment," Proceedings of International Conference on Microwave, RADAR, and Wireless Communications (MIKON2004), vol. 1, pp. 155-158, May 2004

2. S. Kumar, A. Mohammadi, and D. Klymyshyn, "A direct 64QAM modulator suitable for MMIC applications," Microwave Journal, vol. 40, pp. 116-122, Apr. 1997

3. R. A. Minasian, "Photonic signal processing of microwave signals," IEEE Trans. Microwave Theory Tech., vol. 54 pp. 832-846, Feb. 2006

4. L. V. T. Nguyen, and D. B. Hunter, "A photonic technique for microwave frequency measurement," IEEE Photon. Tech. Lett., vol. 18, pp. 1188-1190, May 2006

5. J. Capmany, J. Mora, B. Ortega, and D. Pastor, "Microwave photonic filters using low-cost sources featuring tunability, reconfigurability and negative coefficients," Opt. Express., vol. 13, pp. 1412-1417, Mar. 2005

6. H. Emami, N. Sarkhosh, L. A. Bui, and A. Mitchell, "Wideband RF photonic in-phase and quadrature-phase generation," Opt. Lett., vol. 33, pp. 98-100, Jan 2008 